\section{CONVENTIONAL CARDIAC RISK FACTORS ASSOCIATED WITH TRASTUZUMAB-INDUCED CARDIOTOXICITY IN BREAST CANCER: A META-ANALYSIS}

${ }^{1}$ Ramesh Nadarajah*, 'Georgios Koulaouzidis, ${ }^{2}$ Dafni Charisopoulou, ${ }^{3}$ Diana Yung, ${ }^{4}$ Amanda Yung. ${ }^{1}$ Mid Yorkshire NHS Trust; ${ }^{2}$ lppokrateio Hospital of Thessaloniki, Thessaloniki, Greece; ${ }^{3}$ Centre for Liver and Digestive Disorders, Royal Infirmary of Edinburgh; ${ }^{4}$ Faculty of Science, University of Sydney, Australia

\subsection{6/heartjnl-2019-BCS.84}

Introduction Trastuzumab reduces the risk of relapse and improves prognosis for breast cancer patients with expression of human epidermal growth factor receptor 2 (HER-2). However, it has proven to be associated with cardiotoxicity, manifest as an asymptomatic decrease in Left Ventricular Ejection Fraction (LVEF) and less often as clinical heart failure (HF). Published studies have tried to identify risk factors predisposing to cardiotoxicity but the results are not uniform. The aim of this meta-analysis is to identify the association of conventional cardiovascular risk factors with the development of Trastuzumab-induced cardiotoxicity (TIC).

Methods A literature search of PubMed was conducted to identify studies examining the association between cardiovascular risk factors and TIC as defined by clinical HF or deterioration in LVEF [Figure 1]. Data were extracted and pooled odds ratios (ORs) with 95\% confidence intervals (CIs) were calculated to examine the odds of developing TIC for each of the risk factors. Results A total of 35 studies met our criteria and were included in the analysis. Age 60 years old (OR: 2.03; 95\% CI: $1.38-3.00 ; \mathrm{p}=0.0004$ ), hypertension (OR: 2.01; 95\% CI: $1.30-3.09 ; \mathrm{p}=0.002$ ), smoking (OR: $1.33 ; 95 \% \mathrm{CI}$ : $1.07-1.65 ; \mathrm{p}=0.01$ ), diabetes mellitus (OR: 1.49; 95\% CI: $1.22-1.81$; $\mathrm{p}<0.0001)$, known history of coronary artery disease (CAD) (OR: 3.72; 95\% CI: 2.11-6.57; p = 0.0005) and family history of CAD (OR: 5.51; 95\% CI: 1.76-17.25; p $<0.00001)$ were significantly associated with the development of TIC. However, obesity (OR: 2.47; 95\% CI: 0.93-6.55; p $=0.07$ ) and hyperlipidaemia (OR: 1.32, 95\% CI: 0.71-2.46; $\mathrm{p}=0.38)$ did not meet statistical significance for association with development of TIC.

Conclusion Identifying women at risk for TIC has several important potential applications. Clinicians may decide to assess LVEF more frequently for patients at highest risk for

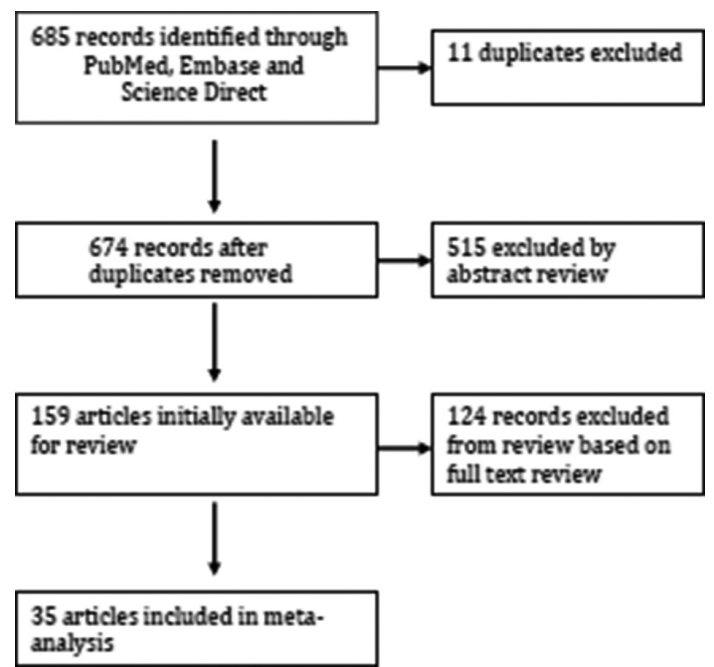

Abstract 86 Figure 1 Flowchart of review design

TIC in order to detect LV systolic dysfunction earlier. Additionally, identifying high-risk patients may play a role for recognition which individuals would obtain the most benefit from prophylactic therapy currently under investigation for preventing TIC. Finally, these risk factors could in the future form the basis of a risk prediction model for TIC.

Conflict of Interest None

\section{DO WE ALWAYS NEED ADVANCED IMAGING TO ASSESS MYOCARDIAL VIABILITY OR IS RESTING ECHOCARDIOGRAPHY ENOUGH IN MOST CASES?}

${ }^{1}$ Sophie Arnold*, ${ }^{2}$ Matthew Ryan, ${ }^{2}$ Haseeb Rahman, ${ }^{3}$ Olga Khaleva, ${ }^{2}$ Divaka Perera. ${ }^{1}$ Guys and St Thomas' NHS Foundation Trust; ${ }^{2}$ King's College London; ${ }^{3}$ Guys and St Thomas' Hospital

\subsection{6/heartjnl-2019-BCS.85}

Background Advanced imaging is a limited resource. Resting transthoracic echocardiography (TTE) is inexpensive and widely available. If TTE identifies clear viable or non-viable myocardium, viability testing could be reserved for "greyzone" cases, with significant resource implications.

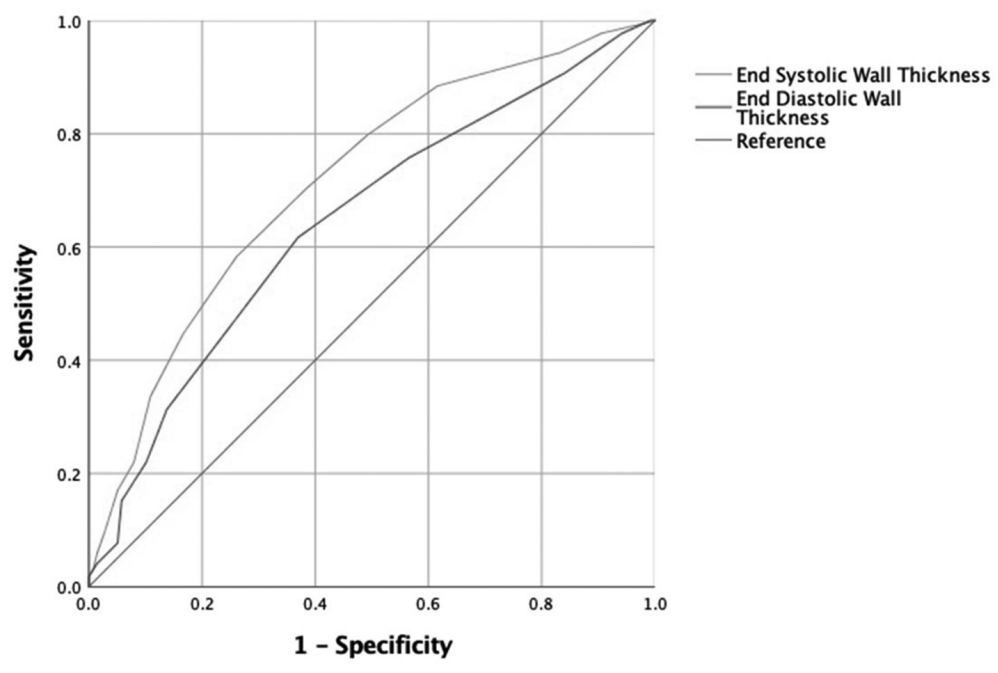

Abstract 87 Figure 1 
Method Adult patients with a clinical diagnosis of ischaemic cardiomyopathy (ICM), known multi-vessel coronary disease and a left ventricular ejection fraction (LVEF) $\leq 40 \%$ who had undergone both cardiac MRI with late gadolinium enhancement (CMR) and TTE were identified from a local database. TTE and MRI images were analysed by experts blinded to clinical and alternative modality information. End systolic wall thickness (ESWT) and end diastolic wall thickness (EDWT) were measured for each LV segment using the AHA 17-segment model. Viability was adjudicated by the standard late gadolinium enhancement transmurality threshold ( $<50 \%$ viable, 50\% nonviable). Receiver operating characteristic curve analysis was performed to determine the accuracy of ESWT and EDWT in predicting CMR-determined viability. See Figure 1.

Results 237 patient records were screened, 44 met inclusion criteria. Nine of these patients were excluded due to poor image quality (7 TTE, 2 CMR). 35 patients were included for final analysis (72 \pm 8 years, 91\% male). LVEF was $27.8 \% \pm$ 6.4. A total of 595 myocardial segments were available for analysis. 585 (98\%) segments were analysed, 447 (75\%) were assessed as viable and $138(23 \%)$ as non-viable.

Both EDWT and ESWT were significantly greater in viable compared to non-viable segments $(7.6 \pm 2.6 \mathrm{~mm}$ vs. $6.3 \pm$ $2.2 \mathrm{~mm}, \mathrm{p}<0.001$ and $6.9 \pm 2.8 \mathrm{~mm}$ vs. $9.3 \pm 3.2 \mathrm{~mm}$, $\mathrm{p}<0.001$ respectively).

The area under the curve (AUC) for EDWT was 0.646, with an optimal cut point of $6.5 \mathrm{~mm}$. ESWT was more accurate (AUC 0.715, optimal cut point $7.5 \mathrm{~mm}$ ). An ESWT $>10.5 \mathrm{~mm}$ had a specificity of $91 \%$ for viability; only $14 \%$ of segments were above this threshold. See Figure 2.

Conclusion ESWT and EDWT have limited accuracy in predicting segmental viability and the majority of segments would remain within the grey-zone. These data do not support the use of TTE as a filter for proceeding to advanced imaging for viability testing.

Conflict of Interest None

\section{RAPID ACCESS HEART FAILURE CLINIC: IMPACT OF A PHYSIOLOGIST-DELIVERED SERVICE IN A UK DISTRICT GENERAL HOSPITAL} ${ }^{1}$ Hannah Sinclair*, ${ }^{2}$ Melanie Ackrill, ${ }^{2}$ Hiliary Holdsworth, ${ }^{2}$ Chris Chase, ${ }^{2}$ Maxine Guillen,
${ }^{2}$ Louise Bowman, ${ }^{2}$ Louisa Collins, ${ }^{2}$ Chris H Critoph, ${ }^{2}$ Amy CJ Pine. ${ }^{1}$ Royal Bournemouth and
Christchurch Hospital; ${ }^{2}$ Royal Bournemouth Hospital

\subsection{6/heartjnl-2019-BCS.86}

Background National Institute of Clinical Excellence (NICE) Chronic Heart Failure Guideline NG106 recommends NTproBNP measurement in patients with suspected heart failure (HF). NTproBNP 400ng/L mandates referral to a rapid access HF clinic (RAHFC). Target wait is determined byNTproBNP concentration (400-2000ng/L 6 weeks and $>2000 \mathrm{ng} / \mathrm{L}$ within 2 weeks).

In our institution, RAHFC referrals have doubled in a year. There is little published national data regarding adherence to NICE waiting targets. Initial service evaluation found that only $47 \%$ of patients were seen within the target waiting time. However, many patients with lower range NT proBNP $(<1000 \mathrm{ng} / \mathrm{L})$ had no significant cardiac pathology and were discharged. It was proposed this lower range NTproBNP population (400- 1000ng/l) could be safely managed in a cardiac physiologist-delivered RAHFC overseen by a HF specialist. Additonal benefits would be to upskill cardiac physiologists, free consultant time for more severe HF patients and improve waiting times.

Aim To introduce a supervised cardiac physiologist-led HF clinic for patients with a NTproBNP 400ng/L but $<1000 \mathrm{ng} / \mathrm{L}$ and monitor outcomes.

Method An electronic form was developed to guide clinical questioning and data collection. The clinic was initially run by 2 cardiac physiologists and a specialist HF fellow. All clinics were overseen by a consultant HF cardiologist. Demographics, NYHA class, NTproBNP concentration, waiting time, final diagnosis, further investigations and echo results were recorded. Outcomes at 3, 6 and 8 months were retrospectively assessed for safety.

Results 34 patients were seen in the physiologist-delivered HF clinic between Jun'18 and Jan'19. The proportion seen within target waiting time rose from $47 \%$ to $70 \%$. Mean age 81 and $50 \%$ female. Mean NTproBNP was $730 \mathrm{ng} / \mathrm{L}$. 26.5\% $(\mathrm{n}=9)$ were diagnosed with HFPEF and 2.9\% $(n=1)$ HFREF. 32.4\% $(n=11)$ had AF or paroxysmal AF. In $41.2 \% \quad(n=14)$ NTproBNP was felt to be a false positive. Diagnoses included: pulmonary hypertension $(\mathrm{n}=1)$, moderate and severe aortic stenosis $(n=2)$, moderate aortic regurgitation $(n=1)$, bradyarrhythmia requiring pacing $(\mathrm{n}=1)$, hypertrophic cardiomyopathy $(n=2)$, atrial tachycardia $(n=1)$, ectopy $(n=2)$. As cardiac physiologists gained experience, they began to review patients independently and time slots were reduced. No adverse events were recorded.

Conclusion Demand for RAHFC is high and NICE waiting times are often difficult to meet with current service provision. A physiologist-delivered HF clinic proved safe, effective and dramatically reduced waiting times. There is a national shortage of cardiac physiologists, and staff retention and recruitment is difficult. Expanding the role and skill set of our cardiac physiologists has proved popular and good for staff morale.

NTproBNP threshold mandating urgent referral to RAHFC for patients with $\mathrm{AF}$ and those of advanced age may require further research and consideration.

Conflict of Interest None Declared

\section{HETEROZYGOUS GLOBAL DELETION OF PLASMA MEMBRANE CALCIUM ATPASE 1 (PMCA1HT) MAY REDUCE CARDIAC REMODELLING AFTER TRANSVERSE AORTIC CONSTRICTION IN A MURINE MODEL}

${ }^{1}$ Tahir Nazir*, ${ }^{2}$ Florence Baudoin, ${ }^{2}$ Min Zi, ${ }^{2}$ Sukhpal Prehar, ${ }^{2}$ Elizabeth Cartwright. ${ }^{1}$ Division of Cardiovascular Sciences, University of Manchester; ${ }^{2}$ University of Manchester

\subsection{6/heartjnl-2019-BCS.87}

Background Cardiovascular diseases remain a major cause of mortality worldwide. PMCA1 is the most prevalent isoform of PMCA in the heart. Our group has previously generated novel PMCA1 mutant mouse lines using Cre/loxP technology. Cardiomyocyte-specific PMCA1 knockout mice (PMCA1cko) develop severe cardiomyopathy with $\mathrm{HF}$ and heterozygote global PMCA1 knockout mice (PMCA1Ht) have elevated blood pressure. However, whether global heterozygous deletion of PMCA1Ht has any impact on cardiac remodelling after pathological stress resulting from pressure overload 